\title{
Single Particle Excitation Spectrum of a Proximized Quantum Dot: The Flow Equation Study
}

\author{
M. ZAPALSKA AND T. DOMAŃSKI
}

Institute of Physics, M. Curie-Skłodowska University, pl. M. Curie-Skłodowskiej 1, 20-031 Lublin, Poland

We study the in-gap states of a quantum dot hybridized with the metallic and superconducting reservoirs applying the continuous unitary transformation to the Anderson-type Hamiltonian. We derive the set of flow equations and analyse the effective single particle excitation spectrum of the correlated quantum dot in presence of the induced electron pairing.

DOI: 10.12693/APhysPolA.126.A-137

PACS: 05.10.Cc, 71.27.+a, 72.15.Qm, 74.45.+c

\section{Introduction}

When a nonsuperconducting quantum dot (QD) with discrete energy spectrum is placed in electrical contact with BCS superconductor, then the proximity effect induces the eigenstates whose energies appear in a sub-gap regime. These Andreev bound states (ABS) can be expressed in form of the BCS-like superposition $u|0\rangle-v|\uparrow \downarrow\rangle$ involving an empty $|0\rangle$ and doubly occupied $|\uparrow \downarrow\rangle$ configurations. The other single occupied states $|\sigma\rangle$ are not affected by the BCS condensate and represent true eigenstates of the QD, forming a spin-degenerate doublet.

Recent experiments with use of the self assembled quantum dots [1], semiconducting nanowires [2,3] and carbon nanotubes $[4,5]$ coupled between the superconducting and conducting electrodes clearly indicated appearance of the ABS. Such Andreev states activate an anomalous tunneling channel which operates even if external voltage is below the energy gap threshold $|e V| \leq$ $\Delta$. The in-gap resonant states have been also detected in the quantum dots placed between both superconducting reservoirs [6-8] leading to inversion of the Josephson current.

In what follows we introduce the microscopic model capturing the essential physics of ABS. Next, we design the continuous canonical transformation, derive the set of flow equations and obtain the effective single particle spectral function.

\section{The model}

For description of the ABS we use the Anderson-type quantum impurity model

$$
\begin{aligned}
\hat{H} & =\sum_{\boldsymbol{k} \sigma} \xi_{\boldsymbol{k}} \hat{c}_{\boldsymbol{k} \sigma}^{\dagger} \hat{c}_{\boldsymbol{k} \sigma}+\sum_{\sigma} \varepsilon_{\boldsymbol{d}} \hat{d}_{\sigma}^{\dagger} \hat{d}_{\sigma}-\Delta_{\boldsymbol{d}}\left(\hat{d}_{\uparrow}^{\dagger} \hat{d}_{\downarrow}^{\dagger}+\hat{d}_{\downarrow} \hat{d}_{\uparrow}\right) \\
& +U \hat{n}_{\boldsymbol{d} \uparrow} \hat{n}_{\boldsymbol{d} \downarrow}+\frac{1}{\sqrt{N}} \sum_{\boldsymbol{k} \sigma} V_{\boldsymbol{k}}\left(\hat{c}_{\boldsymbol{k} \sigma}^{\dagger} \hat{d}_{\sigma}+\hat{d}_{\sigma}^{\dagger} \hat{c}_{\boldsymbol{k} \sigma}\right)
\end{aligned}
$$

The localized QD electrons are described by the annihilation (creation) operators $\hat{d}_{\sigma}\left(\hat{d}_{\sigma}^{\dagger}\right)$, where $\sigma$ denotes the spin, $\varepsilon_{\boldsymbol{d}}$ is the energy level and $\Delta_{\boldsymbol{d}}=\min \left\{\Delta, \Gamma_{S} / 2\right\}$ [9] is the on-dot pairing gap. The Coulomb potential $U_{\boldsymbol{d}}$ is responsible for the charging effect and, at sufficiently low temperatures, can lead to the Kondo-type correlations. Another important ingredient is the hybridization $V_{\boldsymbol{k}}$ of the QD with external metallic lead. Energies of the itinerant electrons $\xi_{k}=\varepsilon_{\boldsymbol{k}}-\mu$ are measured with respect to the chemical potentials $\mu$. In this work we shall investigate the low energy features, therefore we assume the wide band limit $\left|V_{\boldsymbol{k}}\right| \ll D$ (where $-D \leq \varepsilon_{\boldsymbol{k}} \leq D$ ).

\section{The method}

We apply the continuous unitary transformation (CUT) originating from the renormalization group treatments. It has been introduced in 1994 independently by Wegner [10] and Wilson with Glazek [11] and proved to be useful approach for studying a number of problems in the condensed matter physics [12]. The so-called flow equation scheme involves unconventional scaling and operates in the full Hilbert space, so that we keep information about all energy scales of the system. This aspect is particularly useful for determining the correlation functions and for studying mutual feedback effects between the large and small energy scales. The CUT algorithm is based on a continuous diagonalization of the Hamiltonian, which is ultimately reduced to a diagonal (or blockdiagonal) structure via the set of infinitesimal transformations. The unitary flow of the Hamiltonian is generated by the anti-Hermitian operator.

Let us briefly sketch the main idea of CUT considering an arbitrary Hamiltonian $\hat{H}=\hat{H}_{0}+\hat{V}$ with the main part $\hat{H}_{0}$ and the off-diagonal contribution $\hat{V}$ (i.e. interactions, external perturbations, etc). Construction of an effective Hamiltonian $\hat{H}(l)=\hat{\mathcal{U}}^{\dagger}(l) \hat{H} \hat{\mathcal{U}}(l)$ is achieved in a sequence of infinitesimal steps, upon varying a continuous flow parameter $l$. Transformation of the Hamiltonian is governed by the differential equation $\frac{\mathrm{d} \hat{H}(l)}{\mathrm{d} l}=[\hat{\eta}(l), \hat{H}(l)]$, where $\hat{\eta}(l) \equiv \frac{\mathrm{d} \hat{\mathcal{U}}(l)}{\mathrm{d} l} \hat{\mathcal{U}}^{-1}(l)$ is the generating operator. It has been proved by Wegner [10] that choosing

$$
\hat{\eta}(l)=[\hat{H}(l), \hat{V}(l)]
$$

guarantees that $\hat{V}(l)$ vanishes in the asymptotic limit $l \rightarrow \infty$. All $l$-dependent observables obey the differential equation

$$
\frac{\mathrm{d} \hat{O} l)}{\mathrm{d} l}=[\hat{\eta}(l), \hat{O}(l)],
$$

similar to the flow equation for the model Hamiltonian.

\section{Effective Hamiltonian}


The Anderson-type Hamiltonian (1) is a microscopic model for the systems exhibiting the Kondo effect. To investigate the Kondo-type correlations we eliminate the hybridization between the proximized quantum dot and the normal electrode

$$
\hat{V}=\frac{1}{\sqrt{N}} \sum_{\boldsymbol{k} \sigma} V_{\boldsymbol{k}}\left(\hat{c}_{\boldsymbol{k} \sigma}^{\dagger} \hat{d}_{\sigma}+\hat{d}_{\sigma}^{\dagger} \hat{c}_{\boldsymbol{k} \sigma}\right) .
$$

The effective Hamiltonian takes the following form [13]:

$$
\begin{aligned}
& \hat{H}(l)=\sum_{\boldsymbol{k} \sigma} \xi_{\boldsymbol{k}}(l) \hat{c}_{\boldsymbol{k} \sigma}^{\dagger} \hat{c}_{\boldsymbol{k} \sigma}+\sum_{\sigma} \varepsilon_{\boldsymbol{d}}(l) \hat{d}_{\sigma}^{\dagger} \hat{d}_{\sigma} \hat{V(l)} \\
& +U(l) \hat{n}_{\boldsymbol{d} \uparrow} \hat{n}_{\boldsymbol{d} \downarrow}-\Delta_{\boldsymbol{d}}(l)\left(\hat{d}_{\uparrow}^{\dagger} \hat{d}_{\downarrow}^{\dagger}+\hat{d}_{\downarrow} \hat{d}_{\uparrow}\right) \\
& \quad-\sum_{\boldsymbol{k}, \boldsymbol{p}, \sigma_{1}, \sigma_{2}} J_{\boldsymbol{k} p}(l) \hat{\boldsymbol{s}}_{\boldsymbol{d}} \cdot \hat{\boldsymbol{S}}_{\boldsymbol{k p} \boldsymbol{p}}
\end{aligned}
$$

where $\hat{\boldsymbol{s}}_{\boldsymbol{d}}$ is the spin operator of QD electrons and $\hat{\boldsymbol{S}}_{\boldsymbol{k}, \boldsymbol{p}}$ describes the spin operator of itinerant electrons. This additional term is important, because it yields the induced spin-spin interaction.

To eliminate the hybridization term (4) we apply the original Wegner prescription (2) to the Hamiltonian (1). We obtain the following anti-Hermitian operator [13]:

$$
\begin{aligned}
\hat{\eta}= & \sum_{\boldsymbol{k} \sigma} \eta_{\boldsymbol{k}}\left(\hat{c}_{\boldsymbol{k} \sigma}^{\dagger} \hat{d}_{\sigma}-\hat{d}_{\sigma}^{\dagger} \hat{c}_{\boldsymbol{k} \sigma}\right)+\sum_{\boldsymbol{k} p \sigma} \eta_{\boldsymbol{k} p}\left(\hat{c}_{\boldsymbol{k} \sigma}^{\dagger} \hat{c}_{\boldsymbol{p} \sigma}-\hat{c}_{\boldsymbol{p} \sigma}^{\dagger} \hat{c}_{\boldsymbol{k} \sigma}\right) \\
& +\sum_{\boldsymbol{k}} \eta_{\boldsymbol{k}}^{(1)}\left(\hat{c}_{\boldsymbol{k} \uparrow}^{\dagger} \hat{d}_{\downarrow}^{\dagger}-\hat{d}_{\downarrow} \hat{c}_{\boldsymbol{k} \uparrow}\right)-\sum_{\boldsymbol{k}} \eta_{\boldsymbol{k}}^{(1)}\left(\hat{c}_{\boldsymbol{k} \downarrow}^{\dagger} \hat{d}_{\uparrow}^{\dagger}-\hat{d}_{\uparrow} \hat{c}_{\boldsymbol{k} \downarrow}\right) \\
& +\sum_{\boldsymbol{k} \sigma} \eta_{\boldsymbol{k}}^{(2)}\left(\hat{c}_{\boldsymbol{k} \sigma}^{\dagger} \hat{d}_{-\sigma}^{\dagger} \hat{d}_{-\sigma} \hat{d}_{\sigma}-\hat{d}_{\sigma}^{\dagger} \hat{d}_{-\sigma}^{\dagger} \hat{d}_{-\sigma} \hat{c}_{\boldsymbol{k} \sigma}\right),
\end{aligned}
$$

where $l$-dependent amplitudes are given by $\eta_{\boldsymbol{k}}(l)=$ $\frac{1}{\sqrt{N}}\left(\xi_{\boldsymbol{k}}(l)-\varepsilon_{\boldsymbol{d}}(l)\right) V_{\boldsymbol{k}}(l), \eta_{\boldsymbol{k} p}(l)=\frac{1}{N} V_{\boldsymbol{k}}(l) V_{\boldsymbol{p}}(1), \eta_{\boldsymbol{k}}^{(1)}(l)=$ $\frac{1}{\sqrt{N}} \Delta_{\boldsymbol{d}}(l) V_{\boldsymbol{k}}(l)$ and $\eta_{\boldsymbol{k}}^{(2)}(l)=-\frac{1}{\sqrt{N}} U(l) V_{\boldsymbol{k}}(l)$.

Transformation of the Hamiltonian proceeds according to the flow equation

$$
\frac{\mathrm{d} \hat{H}(l)}{\mathrm{d} l}=[\hat{\eta}(l), \hat{H}(l)] .
$$

During the flow many additional couplings are generated which did not occur in the initial Hamiltonian. We either linearize such new terms and/or neglect some of the irrelevant terms [14]. From (7) we finally derive the set of coupled flow equations for all $l$-dependent coefficients and solve it numerically. Differences between the renormalized $(l=\infty)$ and initial $(l=0)$ values of $\varepsilon_{\boldsymbol{d}}(l), U(l), \Delta_{\boldsymbol{d}}(l)$ range between 5 to 12 percent, whereas the hybridization $V_{\boldsymbol{k}}(l)$ completetly vanishes for $l \rightarrow \infty$.

From our analysis [13] we find the destructive influence of pairing on the antiferromagnetic order. These theoretical results are in a qualitative agreement with experimental data [2]. Since the effective exchange coupling $J_{\boldsymbol{k} p}$ is suppressed by the induced on-dot pairing $\Delta_{\boldsymbol{d}}$ it further suppresses also the Kondo temperature $T_{\mathrm{K}}$ of the proximized quantum dot.

\section{Spectrum of ABS states}

In this section we analyze the in-gap states of a proximized quantum dot. The excitation spectrum can be determined from the Green function $\left\langle\left\langle\hat{d}_{\uparrow}(0) ; \hat{d}_{\uparrow}^{\dagger}(0)\right\rangle\right\rangle_{\hat{H}(0)}=$ $\left\langle\left\langle\hat{d}_{\uparrow}(l) ; \hat{d}_{\uparrow}^{\dagger}(l)\right\rangle\right\rangle_{\hat{H}(l)}=\left\langle\left\langle\hat{d}_{\uparrow}(\infty) ; \hat{d}_{\uparrow}^{\dagger}(\infty)\right\rangle\right\rangle_{\hat{H}(\infty)}$, where $l$-dependent operators obey the flow Eq. (3). To deduce their evolution we start with the initial derivative

$$
\left(\frac{\mathrm{d}}{\mathrm{d} l} \hat{d}_{\sigma}(l)\right)_{l=0}=\left[\hat{\eta}(0), \hat{d}_{\sigma}(0)\right]
$$

where $\hat{d}_{\sigma}(0)=\hat{d}_{\sigma}$ and $\hat{d}_{\sigma}^{\dagger}(0)=\hat{d}_{\sigma}^{\dagger}$. Using the canonical generator $(6)$ we find

$$
\begin{aligned}
& \frac{\mathrm{d}}{\mathrm{d} l}\left(\hat{d}_{\uparrow}(l)\right)_{l=0}=\sum_{\boldsymbol{k}}\left(\eta_{\boldsymbol{k}}(l) \hat{c}_{\boldsymbol{k} \uparrow}-\eta_{\boldsymbol{k}}^{(1)}(l) \hat{c}_{\boldsymbol{k} \downarrow}^{\dagger}+\eta_{\boldsymbol{k}}^{(2)}(l) \hat{d}_{\downarrow}^{\dagger} \hat{d}_{\downarrow} \hat{c}_{\boldsymbol{k} \uparrow}\right. \\
& \left.\quad+\eta_{\boldsymbol{k}}^{(2)}(l) \hat{d}_{\uparrow} \hat{d}_{\downarrow} \hat{c}_{\boldsymbol{k} \downarrow}^{\dagger}-\eta_{\boldsymbol{k}}^{(2)}(l) \hat{d}_{\downarrow}^{\dagger} \hat{d}_{\uparrow} \hat{c}_{\boldsymbol{k} \downarrow}\right), \\
& \frac{\mathrm{d}}{\mathrm{d} l}\left(\hat{d}_{\downarrow}(l)\right)_{l=0}=\sum_{\boldsymbol{k}}\left(\eta_{\boldsymbol{k}}(l) \hat{c}_{\boldsymbol{k} \downarrow}+\eta_{\boldsymbol{k}}^{(1)}(l) \hat{c}_{\boldsymbol{k} \uparrow}^{\dagger}+\eta_{\boldsymbol{k}}^{(2)}(l) \hat{d}_{\uparrow}^{\dagger} \hat{d}_{\uparrow} \hat{c}_{\boldsymbol{k} \downarrow}\right. \\
& \left.\quad+\eta_{\boldsymbol{k}}^{(2)}(l) \hat{d}_{\downarrow} \hat{d}_{\uparrow} \hat{c}_{\boldsymbol{k} \uparrow}^{\dagger}-\eta_{\boldsymbol{k}}^{(2)}(l) \hat{d}_{\uparrow}^{\dagger} \hat{d}_{\downarrow} \hat{c}_{\boldsymbol{k} \uparrow}\right) .
\end{aligned}
$$

Taking into account the initial operators and the new terms appearing in Eqs. (9),(10) we deduce the following $l$-dependent parameterizations:

$$
\begin{aligned}
& \hat{d}_{\uparrow}(l)=\alpha(l) \hat{d}_{\uparrow}+\beta(l) \hat{d}_{\downarrow}^{\dagger}+\sum_{\boldsymbol{k}}\left(\alpha_{\boldsymbol{k}}(l) \hat{c}_{\boldsymbol{k} \uparrow}+\beta_{\boldsymbol{k}}(l) \hat{c}_{\boldsymbol{k} \downarrow}^{\dagger} \quad(11)\right. \\
& \left.\quad+\gamma_{\boldsymbol{k}}^{(1)}(l) \hat{d}_{\downarrow}^{\dagger} \hat{d}_{\downarrow} \hat{c}_{\mathbf{k} \uparrow}+\gamma_{\boldsymbol{k}}^{(2)}(l) \hat{d}_{\uparrow} \hat{d}_{\downarrow} \hat{c}_{\boldsymbol{k} \downarrow}^{\dagger}+\gamma_{\boldsymbol{k}}^{(3)}(l) \hat{d}_{\downarrow}^{\dagger} \hat{d}_{\uparrow} \hat{c}_{\boldsymbol{k} \downarrow}\right), \\
& \hat{d}_{\downarrow}(l)=\alpha(l) \hat{d}_{\downarrow}-\beta(l) \hat{d}_{\uparrow}^{\dagger}+\sum_{\boldsymbol{k}}\left(\alpha_{\boldsymbol{k}}(l) \hat{c}_{\boldsymbol{k} \downarrow}-\beta_{\boldsymbol{k}}(l) \hat{c}_{\boldsymbol{k} \uparrow}^{\dagger} \quad(12)\right. \\
& \left.\quad+\gamma_{\boldsymbol{k}}^{(1)}(l) \hat{d}_{\uparrow}^{\dagger} \hat{d}_{\uparrow} \hat{c}_{\boldsymbol{k} \downarrow}+\gamma_{\boldsymbol{k}}^{(2)}(l) \hat{d}_{\downarrow} \hat{d}_{\uparrow} \hat{c}_{\boldsymbol{k} \uparrow}^{\dagger}+\gamma_{\boldsymbol{k}}^{(3)}(l) \hat{d}_{\uparrow}^{\dagger} \hat{d}_{\downarrow} \hat{c}_{\boldsymbol{k} \uparrow}\right),
\end{aligned}
$$

with the boundary conditions $\alpha(0)=1$ and $\beta(0)=$ $\alpha_{\boldsymbol{k}}(0)=\beta_{\boldsymbol{k}}(0)=\gamma_{\boldsymbol{k}}^{(1)}(0)=\gamma_{\boldsymbol{k}}^{(2)}(0)=\gamma_{\boldsymbol{k}}^{(3)}(0)=0$. Next, we use the parameterized operators in the flow equation

$$
\frac{\mathrm{d}}{\mathrm{d} l}\left[\hat{d}_{\sigma}(l)\right]=\left[\hat{\eta}(l), \hat{d}_{\sigma}(l)\right]
$$

Comparing the left and the right hand side of Eq. (13) we obtain the following set of differential flow equations for $l$-dependent coefficients:

$$
\begin{aligned}
& \frac{\mathrm{d} \alpha_{\boldsymbol{k}}}{\mathrm{d} l}=\eta_{\boldsymbol{k}} \alpha+2 \sum_{\boldsymbol{p}} \eta_{\boldsymbol{k} p} \alpha_{\boldsymbol{p}}+\eta_{\boldsymbol{k}}^{(1)} \beta+\eta_{\boldsymbol{k}}^{(2)} \beta \chi_{\boldsymbol{d}}^{*}, \\
& \frac{\mathrm{d} \beta_{\boldsymbol{k}}}{\mathrm{d} l}=\eta_{\boldsymbol{k}} \beta+2 \sum_{\boldsymbol{p}} \eta_{\boldsymbol{k} p} \beta_{\boldsymbol{p}}-\eta_{\boldsymbol{k}}^{(1)} \alpha+\eta_{\boldsymbol{k}}^{(2)} \beta n_{\mathbf{d} \uparrow}, \\
& \frac{\mathrm{d} \gamma_{\boldsymbol{k}}^{(1)}}{\mathrm{d} l}=2 \sum_{\boldsymbol{p}} \eta_{\boldsymbol{k} p} \gamma_{\boldsymbol{p}}^{(1)}+\eta_{\boldsymbol{k}}^{(2)} \alpha, \\
& \frac{\mathrm{d} \gamma_{\boldsymbol{k}}^{(2)}}{\mathrm{d} l}=2 \sum_{\boldsymbol{p}} \eta_{\boldsymbol{k} p} \gamma_{\boldsymbol{p}}^{(2)}+\eta_{\boldsymbol{k}}^{(2)} \alpha \\
& \frac{\mathrm{d} \gamma_{\boldsymbol{k}}^{(3)}}{\mathrm{d} l}=2 \sum_{\boldsymbol{p}} \eta_{\boldsymbol{k} p} \gamma_{\boldsymbol{p}}^{(3)}-\eta_{\boldsymbol{k}}^{(2)} \alpha,
\end{aligned}
$$




$$
\begin{aligned}
& \frac{\mathrm{d} \alpha}{\mathrm{d} l}=\sum_{\boldsymbol{k}}\left\{\eta_{\boldsymbol{k}}\left[-\alpha_{\boldsymbol{k}}-\gamma_{\boldsymbol{k}}^{(1)} n_{\boldsymbol{d} \downarrow}+\left(\gamma_{\boldsymbol{k}}^{(2)}+\gamma_{\boldsymbol{k}}^{(3)}\right)\left(n_{\boldsymbol{d} \downarrow}-n_{\boldsymbol{k} \downarrow}\right)\right]+\eta_{\boldsymbol{k}}^{(1)}\left[\beta_{\boldsymbol{k}}+\gamma_{\boldsymbol{k}}^{(3)} \chi_{\boldsymbol{d}}^{*}\right]\right. \\
& \left.\quad+\eta_{\boldsymbol{k}}^{(2)}\left[-\alpha_{\boldsymbol{k}} n_{\boldsymbol{d} \downarrow}+\beta_{\boldsymbol{k}} \chi_{\boldsymbol{d}}^{*}+\gamma_{\boldsymbol{k}}^{(1)} n_{\boldsymbol{d} \downarrow}\left(2 n_{\boldsymbol{k} \uparrow}-1\right)+\gamma_{\boldsymbol{k}}^{(2)} n_{\boldsymbol{k} \downarrow}\left(n_{\boldsymbol{d} \downarrow}-1\right)+\gamma_{\boldsymbol{k}}^{(3)} n_{\boldsymbol{d} \downarrow}\left(1-n_{\boldsymbol{k} \downarrow}\right)\right]\right\}, \\
& \frac{\mathrm{d} \beta}{\mathrm{d} l}=\sum_{\boldsymbol{k}}\left\{\eta_{\boldsymbol{k}}\left[-\beta_{\boldsymbol{k}}+\chi_{\boldsymbol{d}}\left(-\gamma_{\boldsymbol{k}}^{(1)}+\gamma_{\boldsymbol{k}}^{(2)}+\gamma_{\boldsymbol{k}}^{(3)}\right)\right]+\eta_{\boldsymbol{k}}^{(1)}\left[-\alpha_{\boldsymbol{k}}+\gamma_{\boldsymbol{k}}^{(1)}\left(n_{\boldsymbol{k} \uparrow}-1\right)+\gamma_{\boldsymbol{k}}^{(3)}\left(1-n_{\boldsymbol{d} \uparrow}-n_{\boldsymbol{k} \downarrow}\right)\right]\right. \\
& \left.\quad+\eta_{\boldsymbol{k}}^{(2)}\left[-\alpha_{\boldsymbol{k}} \chi_{\boldsymbol{d}}-\beta_{\boldsymbol{k}} n_{\boldsymbol{d} \uparrow}-\gamma_{\boldsymbol{k}}^{(1)} \chi_{\boldsymbol{d}}\left(2 n_{\boldsymbol{k} \uparrow}+1\right)+\gamma_{\boldsymbol{k}}^{(2)} \chi_{\boldsymbol{d}} n_{\boldsymbol{k} \downarrow}+\gamma_{\boldsymbol{k}}^{(3)} \chi_{\boldsymbol{d}}\left(1-n_{\boldsymbol{k} \downarrow}\right)\right]\right\},
\end{aligned}
$$

where $\chi_{\boldsymbol{d}}=\left\langle\hat{d}_{\downarrow} \hat{d}_{\uparrow}\right\rangle, \chi_{\boldsymbol{d}}^{*}=\left\langle\hat{d}_{\uparrow}^{\dagger} \hat{d}_{\downarrow}^{\dagger}\right\rangle, n_{\boldsymbol{d} \sigma}=\left\langle\hat{d}_{\sigma}^{\dagger} \hat{d}_{\sigma}\right\rangle$ and $n_{\boldsymbol{k} \sigma}=\left\langle\hat{c}_{\boldsymbol{k} \sigma}^{\dagger} \hat{c}_{\boldsymbol{k} \sigma}\right\rangle$. We can now calculate the single particle propagator which takes the following explicit form:

$$
\begin{aligned}
& \left\langle\left\langle\hat{d}_{\uparrow} ; \hat{d}_{\uparrow}^{\dagger}\right\rangle\right\rangle=\left(|\alpha|^{2} a u^{2}+|\beta|^{2} a v^{2}+2 \alpha \beta u v a\right) \frac{1}{\omega-\left(\frac{U}{2}+E_{\boldsymbol{d}}\right)}+\left(|\alpha|^{2} a v^{2}+|\beta|^{2} a u^{2}+2 \alpha \beta u v a\right) \frac{1}{\omega+\left(\frac{U}{2}+E_{\boldsymbol{d}}\right)} \\
& +\left(|\alpha|^{2} b v^{2}+|\beta|^{2} b u^{2}-2 \alpha \beta u v b\right) \frac{1}{\omega-\left(\frac{U}{2}-E_{\boldsymbol{d}}\right)}+\left(|\alpha|^{2} b u^{2}+|\beta|^{2} b v^{2}-2 \alpha \beta u v b\right) \frac{1}{\omega+\left(\frac{U}{2}-E_{\boldsymbol{d}}\right)} \\
& +\sum_{\boldsymbol{k}}\left(\left|\alpha_{\boldsymbol{k}}\right|^{2}+2 \alpha_{\boldsymbol{k}} \gamma_{\boldsymbol{k}}^{(1)} n_{\boldsymbol{d} \downarrow}\right) \frac{1}{\omega-\xi_{\boldsymbol{k}}}+\sum_{\boldsymbol{k}}\left(\left|\beta_{\boldsymbol{k}}\right|^{2}-2 \beta_{\boldsymbol{k}} \gamma_{\boldsymbol{k}}^{(2)} \chi_{\boldsymbol{d}}\right) \frac{1}{\omega+\xi_{\boldsymbol{k}}} \\
& +\frac{\tanh \left(\frac{E_{\boldsymbol{d}}}{k_{\mathrm{B}} T}\right)}{1+\frac{\exp \left(\frac{U}{2 k_{\mathrm{B}} T}\right)}{\cosh \left(\frac{E_{\boldsymbol{d}}}{k_{\mathrm{B}} T}\right)}} \sum_{\boldsymbol{k}}\left|\gamma_{\boldsymbol{k}}^{(2)}\right|^{2}\left(|v|^{2} \frac{f_{B E}\left(2 E_{\boldsymbol{d}}\right)+f_{F D}\left(\xi_{\boldsymbol{k}}\right)}{2 E_{\boldsymbol{d}}+\xi_{\boldsymbol{k}}-\omega}+|u|^{2} \frac{f_{B E}\left(-2 E_{\boldsymbol{d}}\right)+f_{F D}\left(\xi_{\boldsymbol{k}}\right)}{-2 E_{\boldsymbol{d}}+\xi_{\boldsymbol{k}}-\omega}\right) .
\end{aligned}
$$

BCS-type factors are defined as $u^{2}=\frac{1}{2}\left[1+\frac{\varepsilon_{\boldsymbol{d}}+\frac{U}{2}}{E_{\boldsymbol{d}}}\right]$, $v^{2}=1-u^{2}$ and the additional spectral weigths by $a=\frac{1+\exp \left(-\frac{E_{\boldsymbol{d}}}{k_{B} T}\right)}{2+2 \cosh \left(\frac{E_{\boldsymbol{d}}}{k_{B} T^{T}}\right)}$ and $b=1-a$. Energies $E_{\boldsymbol{d}}=$ $\sqrt{\left(\varepsilon_{\boldsymbol{d}}+\frac{U}{2}\right)^{2}+\Delta_{\boldsymbol{d}}^{2}}$ of the Andreev quasiparticles are formally expressed by the quantities estimated in the limit $l \rightarrow \infty$.

Spectral function $\rho(\omega)=-\frac{1}{\pi} \Im\left\langle\left\langle\hat{d}_{\uparrow} ; \hat{d}_{\uparrow}^{\dagger}\right\rangle\right\rangle_{\omega+i 0^{+}}$of the correlated and proximized quantum dot consists thus of: (i) four delta peaks (corresponding to the long-lived subgap Andreev states), (ii) a flat background spread all over the entire bandwidth (which comes from the scattering beetween the QD and itinerant electrons), and (iii) two additional (temperature-dependent) features appearing near $\pm 2 E_{\boldsymbol{d}}$. The latter effect is probably caused by the higher order scattering mechanism, involving the subgap Andreev states. A more detailed analysis of this puzzling effect along with the numercial computations of the spectral function shall be discussed elsewhere.

\section{Summary}

We have investigated the spectral properties of the correlated quantum dot affected by the proximity induced electron pairing. Hybridization of the quantum dot to the external bath of mobile electrons has been eliminated by means of the continuous unitary transformation. We have found that effective Hamiltonian has the spin-exchange interactions and their antiferromagnetic magnitude is considerably suppressed by the ondot pairing. The resulting single particle spectral function consists of four subgap peaks, a flat background and some additional many-body features apparently originating from the scattering involving the itinerant electrons and the subgap Andreev quasiparticles. This energy structure seems to be relevant to the singlet-doublet quantum transition observed in the recent experimental measurements [15]. 


\section{References}

[1] R.S. Deacon, Y. Tanaka, A. Oiwa, R. Sakano, K. Yoshida, K. Shibata, K. Hirakawa, S. Tarucha, Phys. Rev. Lett. 104, 076805 (2010); Phys. Rev. B 81, 121308(R) (2010).

[2] E.J.H. Lee, X. Jiang, R. Aguado, G. Katsaros, C.M. Lieber, S. De Franceschi, Phys. Rev. Lett. 109, 186802 (2012).

[3] E.J.H. Lee, X. Jiang, M. Houzet, R. Aguado, Ch.M. Lieber, S. De Franceschi, Nature Nanotechnol. 9, 79 (2014).

[4] J.D. Pillet, P. Joyez, R. Žitko, F.M. Goffman, Phys. Rev. B 88, 045101 (2013).

[5] J. Schindele, A. Baumgartner, R. Maurand, M. Weiss, C. Schönenberger, Phys. Rev. B 89, 045422 (2014).

[6] J.-D. Pillet, C.H.L. Quay, P. Morfin, C. Bena, A. Levy-Yeyati, P. Joyez, Nature Phys. 6, 965 (2010).

[7] T. Dirks, T.L. Hughes, S. Lal, B. Uchoa, Y.-F. Chen, C. Chialvo, P.M. Golbart, N. Mason, Nature Phys. 7, 386 (2011).
[8] L. Bretheau, C.Ö. Girit, H. Pothier, D. Esteve, C. Urbina, Nature 499, 312 (2013).

[9] J. Barański, T. Domański, J. Phys. Condens. Matter 25, 435305 (2013).

[10] F. Wegner, Ann. Phys. (Leipzig) 506, 77 (1994).

[11] S.D. Głazek, K.G. Wilson, Phys. Rev. D 49, 4214 (1994).

[12] S. Kehrein, The Flow Equation Approach to ManyParticle Systems, Springer Tracts in Modern Physics, Vol. 215, Springer, Berlin 2006.

[13] M. Zapalska, T. Domański, arXiv:1402.1291, submitted for publication.

[14] S. Kehrein, A. Mielke, J. Phys. A Math. Gen. 27, 4259 (1994); S. Kehrein, A. Mielke, Ann. Phys. 252, 1 (1996).

[15] R. Maurand, Ch. Schönenberger, Physics 6, 75 (2013). 